\title{
Global Regulation of Gene Expression by the MafR Protein of Enterococcus faecalis
}

\author{
Sofía Ruiz-Cruz', Manuel Espinosa1, Oliver Goldmann² and Alicia Bravo ${ }^{1 *}$ \\ ${ }^{1}$ Centro de Investigaciones Biológicas, Consejo Superior de Investigaciones Científicas, Madrid, Spain, ${ }^{2}$ Infection \\ Immunology Research Group, Helmholtz Centre for Infection Research, Braunschweig, Germany
}

OPEN ACCESS

Edited by:

Eric Altermann,

AgResearch Ltd, New Zealand

Reviewed by:

Elaine Allan,

University College London, UK

Suleyman Yildirim,

Istanbul Medipol University, Turkey

Grzegorz Wegrzyn,

University of Gdañsk, Poland

*Correspondence:

Alicia Bravo

abravo@cib.csic.es

Specialty section: This article was submitted to

Evolutionary and Genomic

Microbiology,

a section of the journal

Frontiers in Microbiology

Received: 02 September 2015 Accepted: 17 December 2015

Published: 11 January 2016

Citation:

Ruiz-Cruz S, Espinosa M, Goldmann O and Bravo A (2016) Global Regulation of Gene Expression by the MafR Protein of Enterococcus faecalis. Front. Microbiol. 6:1521. doi: 10.3389/fmicb.2015.01521
Enterococcus faecalis is a natural inhabitant of the human gastrointestinal tract. However, as an opportunistic pathogen, it is able to colonize other host niches and cause life-threatening infections. Its adaptation to new environments involves global changes in gene expression. The EF3013 gene (here named mafR) of $E$. faecalis strain V583 encodes a protein (MafR, 482 residues) that has sequence similarity to global response regulators of the Mga/AtxA family. The enterococcal OG1RF genome also encodes the MafR protein (gene OG1RF_12293). In this work, we have identified the promoter of the mafR gene using several in vivo approaches. Moreover, we show that MafR influences positively the transcription of many genes on a genome-wide scale. The most significant target genes encode components of PTS-type membrane transporters, components of ABC-type membrane transporters, and proteins involved in the metabolism of carbon sources. Some of these genes were previously reported to be up-regulated during the growth of $E$. faecalis in blood and/or in human urine. Furthermore, we show that a mafR deletion mutant strain induces a significant lower degree of inflammation in the peritoneal cavity of mice, suggesting that enterococcal cells deficient in MafR are less virulent. Our work indicates that MafR is a global transcriptional regulator. It might facilitate the adaptation of $E$. faecalis to particular host niches and, therefore, contribute to its potential virulence.

Keywords: Enterococcus faecalis, global regulators, gene expression, carbon sources, mice peritonitis

\section{INTRODUCTION}

The Gram-positive bacterium Enterococcus faecalis is usually found among the commensal microflora of the human gastrointestinal tract. However, it can become pathogenic and cause a variety of community-acquired and health care-associated infections, such as urinary tract infections, endocarditis, and bacteraemia. At present, E. faecalis is considered one of the most important genetic reservoirs of mobile elements, including those encoding antibiotic resistance (Fisher and Phillips, 2009; Hollenbeck and Rice, 2012). E. faecalis strain V583 was the first vancomycin-resistant clinical isolate reported in the United States. Its genome sequence was published in 2003 and revealed that more than a quarter of the genome consists of probable

Abbreviations: $\mathrm{ABC}$ transporter, ATP-binding cassette transporter; BHI, Brain Heart Infusion; M9YE, M9 minimal medium supplemented with yeast extract; OD, optical density; PRD, PTS regulation domain; PTS, phosphoenolpyruvate:carbohydrate phosphotransferase system. 
mobile or foreign DNA (Paulsen et al., 2003). E. faecalis strain OG1RF is a rifampicin and fusidic acid-resistant derivative of the OG1 human isolate. Its genome sequence was published in 2008 (Bourgogne et al., 2008). Compared to V583, the OG1RF genome contains 227 unique open reading frames but has fewer mobile genetic elements. Both enterococcal strains have contributed to the identification of numerous factors important for virulence (Fisher and Phillips, 2009). However, our understanding of the mechanisms involved in the pathogenicity of E. faecalis is still very limited. In general, global transcriptional regulators that respond to specific external signals are crucial in the adaptation of pathogenic bacteria to different host niches.

The ability to metabolize numerous carbohydrates provides enterococci with an advantage in colonizing competitive environments (Ramsey et al., 2014). The E. faecalis V583 genome encodes pathways for the utilization of more than 15 different sugars (Paulsen et al., 2003). Many of them are substrates for the PTS. E. faecalis V583 has 35 probable PTStype sugar transporters, in addition to other transporters for sugar and polyol utilization, such as the ABC-type systems (ABC transporter family; Paulsen et al., 2003). The PTS not only transports and phosphorylates carbohydrates but also carries out regulatory functions. Some proteins have integrated a specific PTS-recognized phosphorylation domain known as the PRD (Deutscher et al., 2014). PRDs have been found in both transcriptional antiterminators and transcriptional activators that control the expression of genes involved in the uptake and metabolism of carbohydrates.

Frequently, virulence gene regulators sense changes in carbon source availability (Poncet et al., 2009), as it seems to be the case of the Mga and AtxA regulators from Streptococcus pyogenes and Bacillus anthracis, respectively. These proteins are members of an emerging class of PRD-containing global response regulators (Hondorp et al., 2013; Hammerstrom et al., 2015). Mga (530 amino acids) was shown to control the expression of $\sim 10 \%$ of the $S$. pyogenes genome (Ribardo and McIver, 2006). It activates the transcription of numerous genes that enable the bacterium to colonize specific host tissues and evade the host immune response (Ribardo and McIver, 2006; Hondorp and McIver, 2007). Mga is phosphorylated in vivo (Sanson et al., 2015) and can be phosphorylated in vitro by components of the PTS (Hondorp et al., 2013). AtxA (475 amino acids) was reported to have both positive and negative effects on gene expression (Bourgogne et al., 2003). It controls the expression of chromosomal genes, in addition to genes located on the virulence plasmids pXO1 (anthrax toxin genes) and pXO2 (capsule synthesis genes). Some of the AtxA-regulated genes encode secreted proteins and proteins implicated in transcriptional regulation and signaling (Bourgogne et al., 2003). The activity of AtxA is modulated by phosphorylation of histidine residues within PRDs (Tsvetanova et al., 2007). An additional member of the Mga/AtxA family of regulators is likely the MgaSpn protein (493 amino acids), which contributes to the virulence of $S$. pneumoniae (Hemsley et al., 2003; Solano-Collado et al., 2012, 2013). Mga, AtxA, and MgaSpn were predicted to have the same organization of functional domains (Hondorp and McIver, 2007). Microarray experiments showed that MgaSpn influences negatively the expression of several genes located within the $r l r A$ pathogenicity islet (Hemsley et al., 2003). Moreover, it has been shown that MgaSpn activates directly the expression of a four-gene operon of unknown function (Solano-Collado et al., 2012). This regulator binds to DNA with little or no sequence specificity and no consensus DNA target was found (Solano-Collado et al., 2013).

Gene EF3013 (named mafR herein) of the enterococcal V583 strain encodes a 482-amino acids protein (MafR; Mga/AtxAlike faecalis regulator) that has sequence similarity to regulators of the Mga/AtxA family (40.7\% of similarity with AtxA). Although the three-dimensional structure of this protein has been solved by X-ray crystallography (PDB 3SQN; Osipiuk et al., unpublished results), its potential role in global regulation of gene expression has not previously been investigated. In this work, we have addressed this question using genomewide microarrays designed for strain OG1RF. We show that MafR influences positively the transcription of many genes that encode components of PTS transporters, components of ABC transporters, and proteins involved in the metabolism of carbon sources. We also show that MafR has a positive effect on the utilization of glycerol, maltose, and mannitol, suggesting that MafR may facilitate the survival of E. faecalis in particular host niches. Furthermore, our results suggest that a mafR deletion mutant strain is less virulent in a mouse peritonitis model.

\section{MATERIALS AND METHODS}

\section{Oligonucleotides, Bacterial Strains, and Plasmids}

The oligonucleotides used are listed in Tables $\mathbf{1}$ and 2. The E. faecalis strains V583 (Paulsen et al., 2003), OG1RF (Bourgogne et al., 2008), and JH2-2 (Jacob and Hobbs, 1974) were used in this work. To construct the OG1RF $\triangle$ mafR mutant strain, plasmid

TABLE 1 | Oligonucleotides used in the construction of bacterial strains and plasmids, in RT-PCR assays and in primer extension assays.

\begin{tabular}{|c|c|}
\hline Name & Sequence $\left(5^{\prime}\right.$ to $\left.3^{\prime}\right)$ \\
\hline A & GATTAGCTGAATGGTCATCGTGG \\
\hline$B$ & GTTAAAACGTGTGATAACGG \\
\hline C & GACCAATCCCCTIITATCCG \\
\hline $\mathrm{D}$ & AATGAAGAGTGAGCTCTGCTAG \\
\hline UpPma & AGGAATCAGTGGAGCTCCTGTCGGTAA \\
\hline DwPma & GTACATGGCAAGAGCTCCTITGCTT \\
\hline DwPma2 & СTCCTITGCTTAAGAGCTCGGATAAAAAG \\
\hline INTgfp & CATCACCATCTAATTCAACAAG \\
\hline 12292 Nco & CGCAAACCTITCCATGGTCAATACGACGC \\
\hline maClaR & ССTCCTITGCTTAAGAATATCGATAAAAAG \\
\hline maClaF & CAAGAATTAATGATCGATCAAGCCGCAG \\
\hline 12294 Nco & ATAACCAAACGATCCATGGCGAAAGAAAG \\
\hline P2493ClaF & AATAGGTGATCGATTGTTAAATATCTG \\
\hline P2493ClaR & AАCTCTCACATCGATTGAACAAGCATGCAAAATAC \\
\hline maSphF & ПППТTCCGTATTCGCATGCAAAAGGAGG \\
\hline maSphR & AACCAAACGATGCATGCCGAAAGAAAGC \\
\hline
\end{tabular}

Restriction sites are shown in bold. 
TABLE 2 | Oligonucleotides used in qRT-PCR assays.

\begin{tabular}{|c|c|c|}
\hline Locus tag & Forward primer $\left(5^{\prime}\right.$ to $\left.3^{\prime}\right)$ & Reverse primer $\left(5^{\prime}\right.$ to $\left.3^{\prime}\right)$ \\
\hline OG1RF_10298 & GGCGTTCATTACGTTGCTGA & TCGCTTCGTCAATGGTTTGAT \\
\hline OG1RF_10456 & AGACGCCAATITGTTAGAACG & CACAACTAGCGGCTAAAGAAG \\
\hline OG1RF_10683 & CAGAAGATGGCTTACACATTACCT & GAGTAGGCTGTCCATGTCGCT \\
\hline OG1RF_10684 & TAATGGTCGTTGTGGCAGTA & AATTGCCCAACCGATACTCT \\
\hline OG1RF_11135 & CGTTCGTAGTITGCTGTCA & GAAGGGACAAAGCCGATTCT \\
\hline OG1RF_11146 & GAGGGTGGCTाAGTGGAGA & ТTCACCTTCTGCTACGACTT \\
\hline OG1RF_11592 & AACAAGCCGCCTTATTGGT & GGTTCTTCGCCAGTGTTCAT \\
\hline OG1RF_11763 & AACATCGGCGGTATCTTCAG & ATGCCTACATCACCAGTAGC \\
\hline OG1RF_11948 & CTTGTACTGATGTGACTGGGTT & CCAACGCTCCTGTAATGGTT \\
\hline OG1RF_12398 & CCCGACGAATGATITGCCTA & ATCTAACGAACCAGCGACACT \\
\hline OG1RF_12405 & GAAGCATTGCGTITGGAGAT & AGAAGCGACCACTाTGTाTG \\
\hline OG1RF_12439 & GCAACGAAATGGTGGAACAG & AAGGCATCGGCAATCTCTAAG \\
\hline OG1RF_12564 & ACTTGTTCGTGGACGGATTC & TGCAATGCCAACTTCTGTTA \\
\hline
\end{tabular}

pBVGh (thermosensitive replicon) was used (Blancato and Magni, 2010). To this end, a 560-bp region located upstream of the mafR gene (locus_tag OG1RF_12293) was amplified by PCR using primers $12292 \mathrm{Nco}$ and $\mathrm{maClaR}$. Also, a 494-bp region that includes the $3^{\prime}$-end of mafR was amplified using primers maClaF and 12294Nco. Both PCR-synthesized DNAs were digested with ClaI, mixed in equimolecular amounts and ligated with T4 DNA ligase. The ligation mixture was then used as template for PCR amplification using primers 12292 Nco and 12294 Nco. The 1017bp PCR product was digested with NcoI, and the restriction fragment was cloned into the NcoI site of plasmid pBVGh (plasmid pBVG $\Delta m a f R$ ). Strain OG1RF harboring pBVG $\Delta m a f R$ was used to generate strain OG1RF $\Delta$ mafR following the protocol (integration/excision process) reported by Blancato and Magni (2010). Dye terminator sequencing at Secugen (CIB, Madrid) confirmed that OG1RF $\triangle$ mafR lacks the chromosomal region that spans coordinates 2421575 to 2422640 . A similar procedure was used for the construction of strain JH2-2 $\Delta$ mafR. Plasmids pAS (terminator-probe vector) and pAST (promoter-probe vector) were described (Ruiz-Cruz et al., 2010). They carry the tet $L$ gene (tetracycline resistance). To construct pAST-Pma, a 242bp region of the enterococcal V583 genome was amplified by PCR using the UpPma and DwPma primers. The amplified DNA was digested with SacI, and the 215-bp digestion product (coordinates 2888864-2889078) was inserted into the SacI site of pAST. In pAST-Pma, $g f p$ expression is under the control of the Pma promoter. To construct pAST-Pma 19 and pAS$P m a \Delta 19$, a 228-bp region of the V583 genome was amplified with the UpPma and DwPma2 primers. After SacI digestion, the 196-bp restriction fragment (coordinates 2888864-2889059) was cloned into the SacI site of both pAST (pAST-Pma 19 ) and pAS (pAS-Pma 19 ). The expression vector pDLF (this work) is based on the pDL287 plasmid (LeBlanc et al., 1993), which carries a kanamycin resistance gene. pDLF has an engineered unique restriction site for $S p h I$ downstream of the enterococcal P2493 promoter (Ruiz-Cruz et al., 2010). For its construction, a 194-bp region of the V583 genome, which contains promoter P2493, was amplified by PCR using primers P2493ClaF and P2493ClaR. The PCR product was digested with ClaI, and the 171-bp restriction fragment was ligated to the ClaI-linearized pDL287. Plasmid pDLFmafR carries the P2493::mafR fusion gene. For its construction, a 1546-bp region of the OG1RF genome was amplified using the maSphF and maSphR primers. After SphI digestion, the 1514-bp restriction fragment was inserted into the SphI site of pDLF.

\section{Growth and Transformation of Bacteria}

Enterococcus faecalis was routinely grown in BHI medium, which was supplemented with tetracycline $(4 \mu \mathrm{g} / \mathrm{ml})$ when the cells harbored a plasmid based on pAS or pAST, and with kanamycin $(250 \mu \mathrm{g} / \mathrm{ml})$ when the plasmid was based on pDLF. Enterococcal cells containing plasmid pBVG $\Delta$ mafR were grown in tryptone-yeast extract broth supplemented with erythromycin $(5 \mu \mathrm{g} / \mathrm{ml})$. Experiments were performed at $37^{\circ} \mathrm{C}$ without aeration. To analyze the effect of MafR on the utilization of different carbon sources, bacteria were grown in M9 minimal medium supplemented with $0.5 \%$ yeast extract (M9YE medium) and the indicated carbon source ( $1 \%$ glycerol, $0.5 \%$ maltose, or $0.5 \%$ mannitol). When glycerol was used as carbon source, the medium was also supplemented with $0.26 \%$ fumarate. In these assays, 96-well microplates were used. BHI cell cultures (mid-log phase) were diluted 1:1000 in M9YE medium supplemented or not with the corresponding carbon source, and $200 \mu \mathrm{l} /$ well was applied. Microplates were incubated at $37^{\circ} \mathrm{C}$ without shaking in a Thermo Scientific Varioskan Flash instrument. The bacterial growth was measured at $600 \mathrm{~nm}$ in $15 \mathrm{~min}$ cycle. Before every measurement the microplate was shaken for $10 \mathrm{~s}$. The protocol used to transform E. faecalis by electroporation was described (Shepard and Gilmore, 1995).

\section{DNA Isolation}

For large-scale preparations of chromosomal DNA, enterococcal cells were grown in BHI medium supplemented with $1.25 \%$ glycine. The procedure used was reported previously (Ruiz-Cruz et al., 2010). For small-scale preparations of chromosomal DNA, the Bacterial Genomic Isolation Kit (Norgen Biotek Corporation) was used. For small-scale preparations of plasmid DNA, the High Pure Plasmid Isolation Kit (Roche Applied Science) was used. 
The Suspension Buffer of this kit was supplemented with $50 \mathrm{mM}$ glucose, $1.2 \mathrm{mg} / \mathrm{ml}$ lysozyme, and $240 \mathrm{units} / \mathrm{ml}$ mutanolysin. The Lysis Buffer (0.17 M NaOH, 1\% SDS) was freshly prepared.

\section{Polymerase Chain Reaction (PCR)}

The Phusion High-Fidelity DNA polymerase (Finnzymes) and the Phusion HF buffer were used. Reaction mixtures $(50 \mu \mathrm{l})$ contained 5-30 ng of template DNA, 50 pmol of each primer, $200 \mu \mathrm{M}$ each deoxynucleoside triphosphate (dNTP), and one unit of DNA polymerase. PCR conditions were reported previously (Ruiz-Cruz et al., 2010). PCR products were purified with the QIAquick PCR purification kit (Qiagen).

\section{RNA Isolation}

For primer extension and reverse transcription-PCR (RT-PCR) assays, total RNA was isolated using the Aurum Total RNA Mini Kit (Bio-Rad). Cells were grown to an $\mathrm{OD}$ at $650 \mathrm{~nm}\left(\mathrm{OD}_{650}\right)$ of 0.5 . For microarray and qRT-PCR studies, total RNA was isolated using the RNeasy mini Kit (QIAGEN). Cells were grown to an $\mathrm{OD}_{650}$ of 0.4 . In both cases, cultures were processed as specified by the suppliers, except that cells were resuspended in buffer L (10 mM Tris-HCl, pH 8.0, $1 \mathrm{mM}$ EDTA, $1 \mathrm{mg} / \mathrm{ml}$ lysozyme, 160 units $/ \mathrm{ml}$ mutanolysin) and incubated at $37^{\circ} \mathrm{C}$ for $10 \mathrm{~min}$. An additional DNase I digestion step was performed. The integrity of rRNAs was checked by agarose gel electrophoresis. RNA concentration was determined using a NanoDrop ND-1000 Spectrophotometer.

\section{Primer Extension}

The ThermoScript Reverse Transcriptase enzyme (Invitrogen) and $\left[\alpha-{ }^{32} \mathrm{P}\right]$-dATP $(3000 \mathrm{Ci} / \mathrm{mmol}$; Perkin Elmer) were used. The reaction mixture $(20 \mu \mathrm{l})$ contained $4.5 \mu \mathrm{g}$ of total RNA and $20 \mathrm{pmol}$ of primer. It was incubated at $55^{\circ} \mathrm{C}$ for $45 \mathrm{~min}$. After heating at $85^{\circ} \mathrm{C}$ for $5 \mathrm{~min}$, non-incorporated nucleotides were removed using Illustra MicroSpin ${ }^{\mathrm{TM}}$ G-25 columns (GE Healthcare). Samples were ethanol precipitated and dissolved in loading buffer ( $80 \%$ formamide, $1 \mathrm{mM}$ EDTA, $10 \mathrm{mM} \mathrm{NaOH}$, $0.1 \%$ bromophenol blue, $0.1 \%$ xylene cyanol). cDNA products were analyzed by sequencing gel ( $8 \mathrm{M}$ urea, $6 \%$ polyacrylamide). Dideoxy-mediated chain-termination sequencing reactions using DNA from M13mp18 (Yanisch-Perron et al., 1985) and primer 40 M13 (5'-GTTTTCCCAGTCACGAC-3') were run in the same gel. Labeled products were visualized using a Fujifilm Image Analyzer FLA-3000.

\section{Reverse Transcription-PCR (RT-PCR)}

For first-strand cDNA synthesis, 20 pmol of primer was annealed to $175 \mathrm{ng}$ of total RNA. The mixture was incubated with 15 units of ThermoScript reverse transcriptase (Invitrogen) at $55^{\circ} \mathrm{C}$ for $45 \mathrm{~min}$. PCRs were then carried out using cDNA as the template ( $10 \%$ of the first-strand reaction), and $20 \mathrm{pmol}$ of each primer. To rule out the presence of genomic DNA in the RNA preparation, the same reactions were performed in the absence of the reverse transcriptase. As positive control, PCRs were performed with genomic DNA. PCR products were analyzed by agarose $(0.8 \%)$ gel electrophoresis. Gels were stained with ethidium bromide, and DNA was visualized using a Gel-Doc system (Bio-Rad).

\section{Microarrays}

For each strain under study, total RNA from two biological replicates was isolated. Microarray experiments were performed at Bioarray S.L. (Alicante, Spain). Specific microarrays for strain OG1RF were designed using the web-based application eArray (Agilent). The quality of the RNA preparations was assessed using a TapeStation System and the R6K ScreenTape Kit (Agilent). The Two-Color Microarray-Based Prokaryote Analysis (FairPlay III Labeling) Protocol v.1.3 from Agilent was used. Bioinformatics analysis of the microarray data was done with Bioconductor ${ }^{1}$ using the Limma, Marray, affy, pcaMethods, and EMA packages. The microarray data have been deposited in NCBI's Gene Expression Omnibus (Edgar et al., 2002) and are accessible through GEO Series accession number GSE75409².

\section{Quantitative RT-PCR (qRT-PCR)}

Total RNA from three biological replicates was used. For cDNA synthesis with specific primers (validation of microarray data), the ThermoScript Reverse Transcriptase kit (Invitrogen) was used as described (see "RT-PCR" above), except that the amount of total RNA was $250 \mathrm{ng}$. In addition, the reaction mixtures contained 40 units of RNasin Plus RNase Inhibitor (Promega). Samples were twofold diluted with sterile water and stored at $-80^{\circ} \mathrm{C}$. For cDNA synthesis with random primers (complementation assays), the iScript Select cDNA Synthesis kit (Bio-Rad) was used. Reaction mixtures $(20 \mu \mathrm{l})$ contained $1 \mu \mathrm{g}$ of total RNA, $4 \mu \mathrm{l}$ of iScript Select reaction mix, $2 \mu \mathrm{l}$ of random primers, and $1 \mu \mathrm{l}$ of iScript Reverse Transcriptase. Reactions were incubated at $25^{\circ} \mathrm{C}$ for $5 \mathrm{~min}$, then at $42^{\circ} \mathrm{C}$ for $30 \mathrm{~min}$, and finally at $85^{\circ} \mathrm{C}$ for $5 \mathrm{~min}$. Samples were threefold diluted with sterile water and stored at $-80^{\circ} \mathrm{C}$. To rule out the presence of genomic DNA in the RNA preparations, reactions without adding reverse transcriptase were performed. Quantitative PCRs were carried out using the iQ SYBR Green Supermix (Bio-Rad), and a iCycler Thermal Cycler (Bio-Rad). The reaction mixtures $(20 \mu \mathrm{l})$ contained $1-3 \mu \mathrm{l}$ of cDNA, $10 \mu \mathrm{l}$ of iQ SYBR Green Supermix $2 \mathrm{x}$, and $500 \mathrm{nM}$ of each primer. The initial denaturation step was performed at $95^{\circ} \mathrm{C}$ for $5 \mathrm{~min}$. It was followed by 40 cycles that included the next steps: (i) denaturation at $95^{\circ} \mathrm{C}$ for $30 \mathrm{~s}$; (ii) annealing at 55 or $60^{\circ} \mathrm{C}$ for $30 \mathrm{~s}$; and (iii) extension at $72^{\circ} \mathrm{C}$ for 20 s. Data were analyzed with the $\mathrm{iQ}^{\mathrm{TM}} 5$ Optical System Software. Relative quantification of gene expression was performed using the comparative $C_{\mathrm{T}}$ method (Schmittgen and Livak, 2008). The internal control gene was recA (OG1RF_12439). The threshold cycles values $\left(C_{\mathrm{T}}\right)$ of the genes of interest and the control gene were used to calculate $2^{-\Delta C T}$, where $\Delta C_{\mathrm{T}}=C_{\mathrm{T}}$ gene of interest$C_{\mathrm{T}}$ internal control. For a particular gene, the fold change in expression (FC) between two strains was obtained dividing the corresponding $2^{-\Delta C T}$ values.

\section{Fluorescence Assays}

$\mathrm{JH} 2-2$ cells carrying pAS and pAST derivatives were grown to an $\mathrm{OD}_{650}$ of 0.3 (logarithmic phase). Then, different volumes of the culture (25 $\mu \mathrm{l}$ to $1 \mathrm{ml})$ were centrifuged, and cells were

${ }^{1}$ www.bioconductor.org

${ }^{2}$ https://www.ncbi.nlm.nih.gov/geo/query/acc.cgi?acc=GSE75409 
resuspended in $200 \mu \mathrm{l}$ of PBS buffer $\left(10 \mathrm{mM} \mathrm{Na}_{2} \mathrm{HPO}_{4}, 1 \mathrm{mM}\right.$ $\mathrm{KH}_{2} \mathrm{PO}_{4}, 140 \mathrm{mM} \mathrm{NaCl}, 3 \mathrm{mM} \mathrm{KCl}, \mathrm{pH}$ 7.2). Fluorescence intensity was measured using a Thermo Scientific Varioskan Flash instrument (excitation at $488 \mathrm{~nm}$ and emission at $515 \mathrm{~nm}$ ). In each case, three independent cultures were analyzed. The fluorescence corresponding to $200 \mu \mathrm{l}$ of PBS buffer without cells was around 0.03 arbitrary units.

\section{In Silico Prediction of Intrinsic DNA Curvature}

The curvature propensity plots were calculated with the bend.it server $^{3}$ (Vlahovicek et al., 2003). The intrinsic curvature was calculated as degrees per helical turn $\left(10.5^{\circ} /\right.$ helical turn $=1^{\circ}$ /basepair). The curvature propensity plot was calculated using the consensus scale algorithm (DNase I + nucleosome positioning data) with a windows size of 20-bp.

\section{Infection Model}

Female BALB/c mice (8-10 weeks old) purchased from Harlan Laboratories were used for experimental infection. Mice were infected intraperitoneal with $5 \times 10^{7} \mathrm{CFU}$ of live E. faecalis and euthanized by $\mathrm{CO}_{2}$ inhalation at $24 \mathrm{~h}$ after bacterial inoculation. The infiltrating inflammatory cells were isolated from the site of infection by extensively rinsing with $2 \mathrm{ml}$ of warm DMEM medium. The resulting cell suspension was counted using a Neubauer chamber and levels of inflammatory neutrophils were determined by flow cytometry analysis using anti-mouse Ly6G/C antibody (BD Pharmingen, San Diego, CA, USA). The cell suspension was also used to determine the amount of viable bacteria by plating serial dilutions on bile esculin azide agar (Fluka) plates and the levels of inflammatory cytokines. Mice were housed in a pathogen-free animal facility at the Helmholtz Centre for Infection Research and maintained under standard conditions according to institutional guidelines. Animal experiments were performed in accordance with the German regulations of the Society for Laboratory Animal Science (GVSOLAS) and the European Health Law of the Federation of Laboratory Animal Science Associations (FELASA). All experiments were approved by the ethical board Niedersächsisches Landesamt für Verbraucherschutz und Lebensmittelsicherheit, Oldenburg, Germany (permit 33.942502-04-12/0929).

\section{Cytokine Determination}

The determination of IL-6 levels in the peritoneal lavage was performed by specific enzyme-linked immunosorbent assay (ELISA), using matched antibody pairs and recombinant cytokines as standards. Briefly, 96-well microtiter plates were coated with the corresponding purified anti-murine capture monoclonal anti-IL-6 (Pharmingen, San Jose, CA, USA) at a concentration of $2 \mu \mathrm{g} / \mathrm{ml}$ in sodium bicarbonate buffer overnight at $4^{\circ} \mathrm{C}$. The wells were washed and then blocked with $1 \%$ bovine serum albumin-PBS before the serum samples and the appropriate standard were added to each well.

${ }^{3}$ http://hydra.icgeb.trieste.it/dna/bend_it.html
Biotinylated rat monoclonal anti-IL-6 (BD Pharmingen) at $2 \mu \mathrm{g} / \mathrm{ml}$ was added as the second antibody. Detection was performed with streptavidin-peroxidase, and the plates were developed by use of TMB (3,3',5,5'-tetramethylbiphenyl-4, $4^{\prime}$ diamine).

\section{Statistical Analysis}

Data were analyzed using Prism 5 (GraphPad). Statistical significance was determined by using the unpaired Student's $t$-test for the comparison of two groups. In all analyses, $P<0.05$ was considered statistically significant.

\section{RESULTS AND DISCUSSION}

\section{MafR is a Potential Member of the Mga/AtxA Family of Regulators}

Mga (S. pyogenes), AtxA (B. anthracis), and MgaSpn (S. pneumoniae) belong to a new family of global response regulators involved in virulence (Hondorp et al., 2013; SolanoCollado et al., 2013; Hammerstrom et al., 2015). In the genome of E. faecalis strain V583 (GenBank AE016830.1; Paulsen et al., 2003), the ATG codon at coordinate 2889087 is likely the translation start site of the mafR gene (locus_tag EF3013; Figure 1). It is preceded by a canonical ribosome binding site sequence (AGGAGG). Translation from this ATG codon would generate a protein of 482 residues (MafR), which is a potential transcriptional regulator of the Mga/AtxA family. According to EMBOSS needle global sequence alignment (Rice et al., 2000), MafR has 31.3/19.6, 40.7/22.1, and 38.8/23.7\% of similarity/identity with Mga (530 residues; strain MGAS10394), AtxA (475 residues; strain Ames Ancestor) and MgaSpn (493 residues; strain R6), respectively. Moreover, in the case of MafR, analysis of its amino acid sequence using the Pfam database (Finn et al., 2008) revealed that it has two putative DNA-binding domains within the N-terminal region, the so-called HTH_Mga (Family PF08280, residues 11-69) and Mga (Family PF05043, residues 76-164) domains. Both DNAbinding domains are also present in Mga, AtxA and MgaSpn (Hondorp and McIver, 2007). The three-dimensional structures of MafR (EF3013; PDB 3SQN; Osipiuk et al., unpublished results) and AtxA (PDB 4R6I; Hammerstrom et al., 2015) have been solved, and it has been reported that the closest structural homolog for the AtxA C-terminal region (EIIB-like domain) is the C-terminal region of MafR (Hammerstrom et al., 2015).

\section{Identification of the Pma Promoter}

By RT-PCR experiments (Figure 2), we analyzed the expression of the mafR gene in enterococcal V583 cells. Oligonucleotide A was used for cDNA synthesis. The cDNA products were further amplified by PCR. With oligonucleotides A and B, a PCR product that migrated at the position expected for a 439bp DNA was synthesized. No PCR products were detected using oligonucleotides $\mathrm{A}$ and $\mathrm{C}$, although they were able to amplify a fragment of 498-bp when chromosomal DNA was 


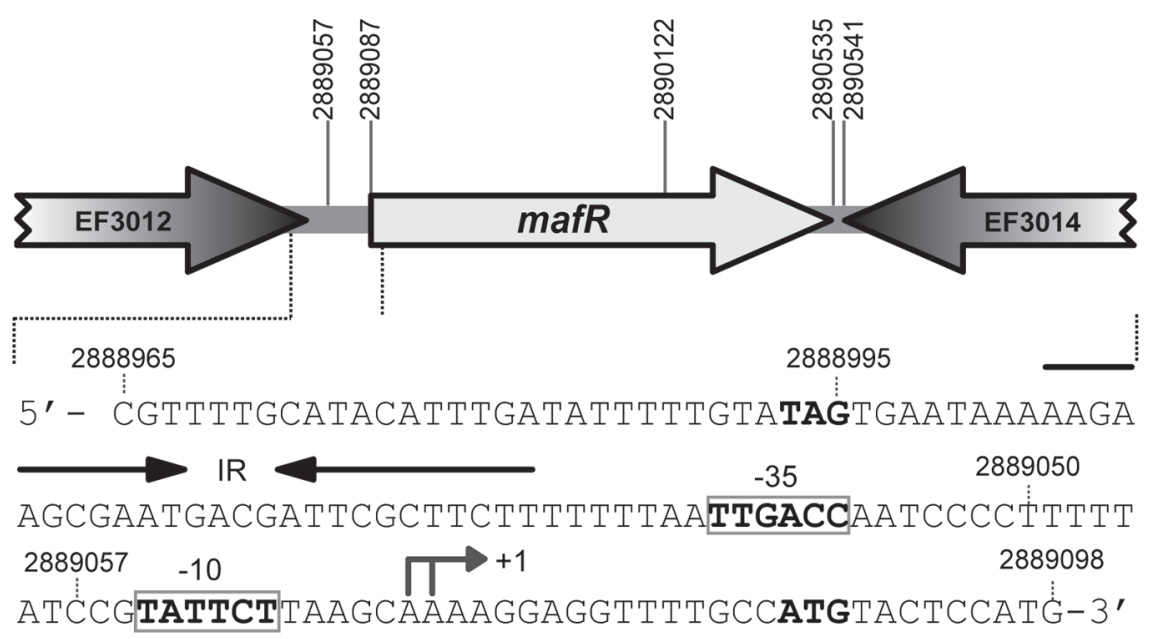

FIGURE 1 | Genetic organization of the mafR (locus_tag EF3013) region in the Enterococcus faecalis V583 genome. The coordinates of the predicted start and stop codons are shown. The nucleotide sequence of the region spanning coordinates 2889098 to 2888965 is shown. The stop codon (TAG) of EF3012 and the start codon (ATG) of mafR are indicated in boldface letters. The transcription start site (+1 position) of the mafR gene, and the main sequence elements ( -35 box and -10 box) of the Pma promoter identified in this work are indicated. IR: inverted-repeat.

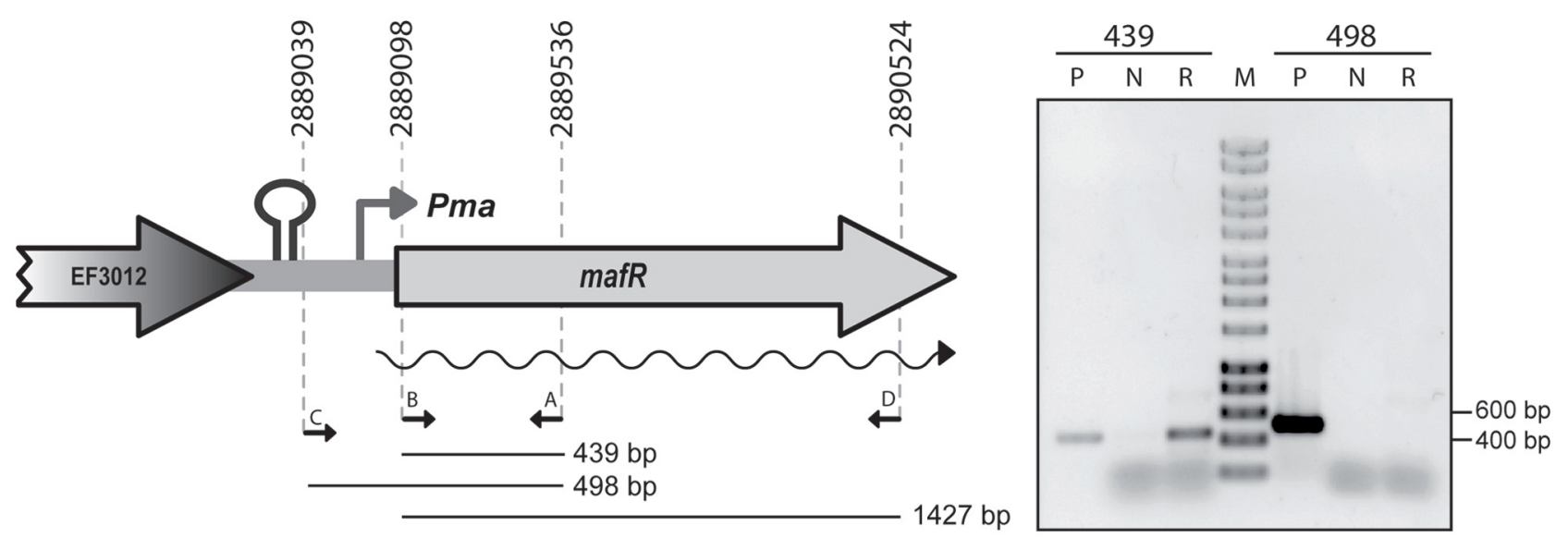

FIGURE 2 | Transcription of mafR in vivo. RT-PCR assays were performed using total RNA from V583 cells. The position of the four primers (A-D) used in the experiment is shown. RT-PCRs samples (lanes R) were analyzed by agarose (0.8\%) gel electrophoresis. The sizes (in bp) of the DNA regions amplified by PCR using chromosomal DNA and the corresponding pair of primers (lanes P; positive control) are indicated. RT-PCRs without adding the reverse transcriptase were also performed (lanes N; negative control). The sizes (in bp) of DNA fragments (lane M) used as molecular weight markers (HyperLadder I, Bioline) are indicated on the right of the gel.

used as template (positive control; Figure 2). We also performed RT-PCR assays using oligonucleotide D for cDNA synthesis. Amplification of the cDNA molecules with oligonucleotides D and B generated a product of 1427-bp (not shown). Thus, transcription of the mafR gene starts at a site located between coordinates 2889039 and 2889098. Next, a 215-bp region of the V583 genome (Pma region; coordinates 2888864 to 2889078) was inserted into the SacI site of the pAST promoter-probe vector (Figure 3A), (Ruiz-Cruz et al., 2010), just upstream of a promoter-less $g f p$ allele that encodes a variant of the green fluorescent protein. The pAST-Pma recombinant plasmid was then introduced into the enterococcal JH2-2 strain. Unlike strain V583 (Paulsen et al., 2003), JH2-2 is a plasmid-free strain
(Jacob and Hobbs, 1974). The fluorescence in cells harboring plasmid pAST-Pma was 2.2-fold higher than in pAST-containing cells (Figure 3A), indicating that the 215-bp Pma region has promoter activity. Sequence analysis of such a region revealed the existence of a putative promoter (Pma) (Figure 1), which shows a $4 / 6$ match at the -10 hexamer $\left(5^{\prime}\right.$-TATTCT- $\left.3^{\prime}\right)$ and a $5 / 6$ match at the -35 hexamer ( $5^{\prime}$-TTGACC- $3^{\prime}$; consensus residues in promoters recognized by a $\sigma$ factor similar to the Escherichia coli $\sigma^{70}$ are shown in bold). Additional experiments confirmed that promoter Pma drives transcription of the $g f p$ gene in plasmid pAST-Pma. Firstly, a deletion of 19 nucleotides that removed the -10 hexamer (Pma $\Delta 19$ region, coordinates 2888864-2889059) reduced the intensity of fluorescence to 


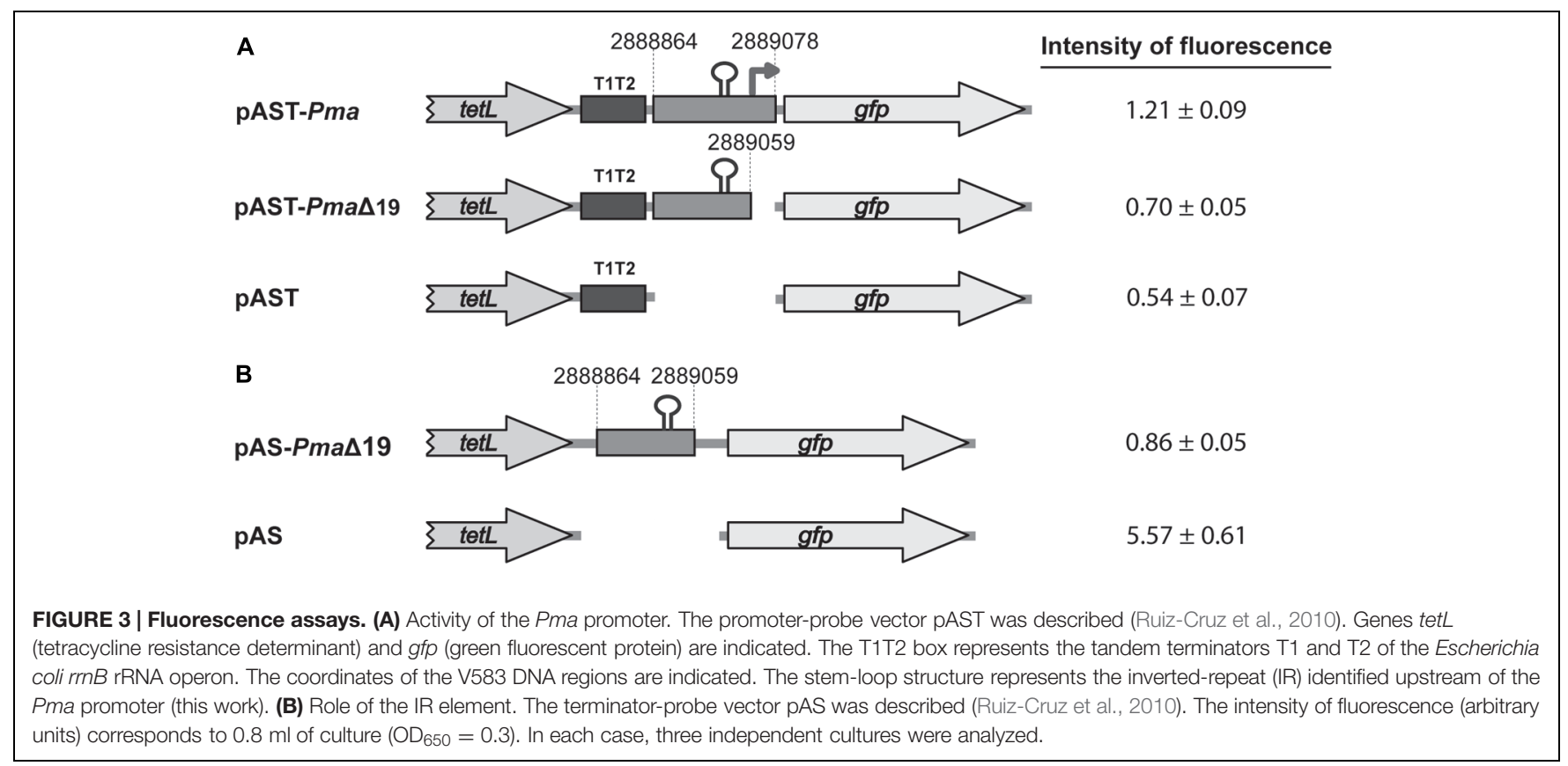

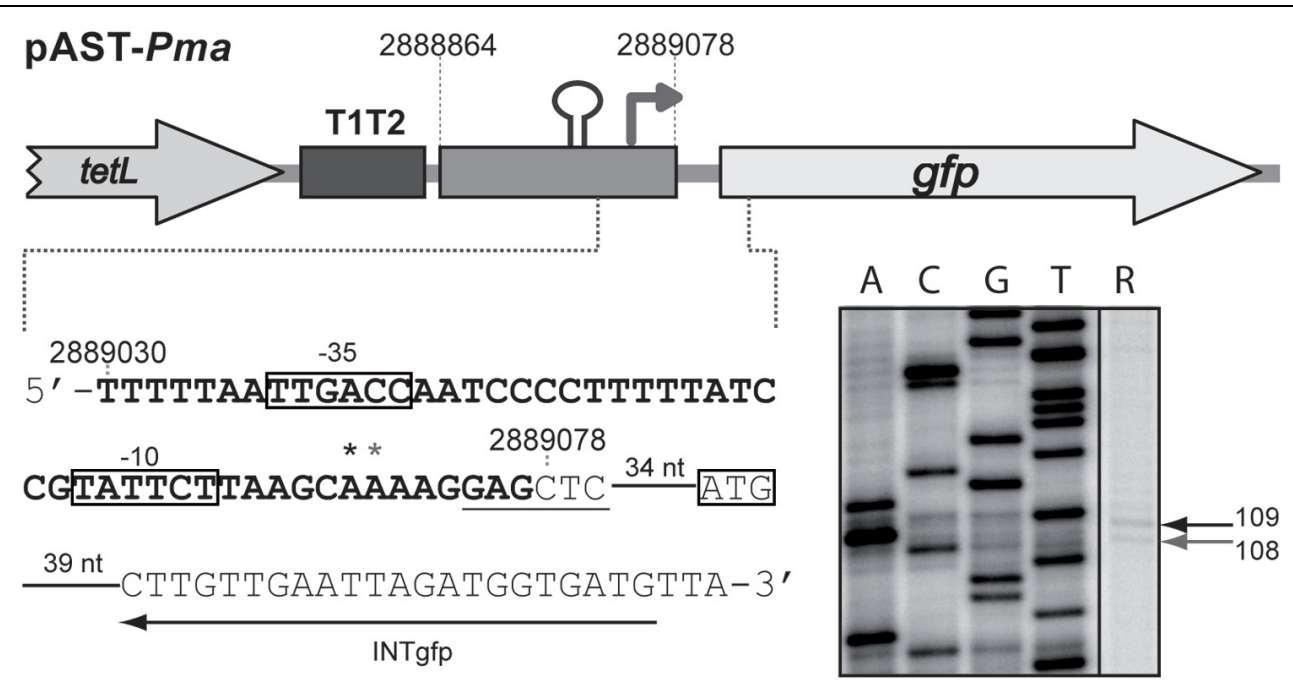

FIGURE 4 | Initiation of transcription at the Pma promoter. Primer extension reactions were carried out using total RNA from JH2-2 cells harboring plasmid pAST-Pma. The main sequence elements of the Pma promoter (-35 and -10 boxes), and the ATG initiation codon of the gfp gene are indicated. The Sacl site is underlined. The asterisks indicate the $3^{\prime}$-end of the cDNA products synthesized using the INTgfp primer. The sizes of the cDNA products (lane R) are indicated in nucleotides on the right of the gel. Dideoxy-mediated chain-termination sequencing reactions (M13mp18 DNA and primer -40 M13) were used as DNA size markers (lanes A, C, G, T).

background levels (cells harboring plasmid pAST-Pma $\Delta 19$ versus cells harboring pAST; Figure 3A). Secondly, two cDNA extension products of 108 and 109 nucleotides were detected using total RNA from cells harboring plasmid pAST-Pma and the INTgfp primer (it anneals to $g f p$ transcripts; Figure 4). Therefore, transcription of the $g f p$ gene starts at the proper distance of 6-7 nucleotides from the -10 element of the Pma promoter.

As shown in Figure 1, sequence analysis of the region located between the TAG stop codon of the locus_tag EF3012 (coordinate 2888995) and the Pma promoter revealed the existence of an inverted-repeat followed by a short stretch of thymine residues (IR element; putative Rho-independent transcriptional terminator; Figure 1). To examine the efficiency of this IR element as transcriptional terminator, we inserted the Pma 19 region (196-bp; coordinates 2888864-2889059) into the pAS terminator-probe vector (Figure 3B), (Ruiz-Cruz et al., 2010). The recombinant plasmid pAS-Pma $\Delta 19$ was introduced into strain JH2-2. In pAS-carrying cells, the promoter-less $g f p$ reporter gene is expressed due to the low efficiency of 
the tetL transcriptional terminator (Ruiz-Cruz et al., 2010). Compared to such cells, the fluorescence was 6.4-fold lower in cells harboring plasmid pAS-Pma 19 (Figure 3B). Thus, there is a transcriptional terminator signal upstream of the Pma promoter.

\section{Deletion of the mafR Gene in Strain OG1RF}

The genome of the enterococcal OG1RF strain has been totally sequenced (GenBank CP002621.1; Bourgogne et al., 2008). The nucleotide sequence of the region that spans coordinates 2888965 to 2889098 in the V583 genome (Figure 1) is identical in the OG1RF genome (coordinates 24214832421616). By RT-PCR assays using oligonucleotides D and $B$ (see Figure 2), we confirmed that the OG1RF mafR gene (locus_tag OG1RF_12293; coordinates 2421605-2423053) is expressed under standard laboratory conditions (BHI broth, $37^{\circ} \mathrm{C}$, without aeration). Compared to strain V583, MafR of the OG1RF strain has five amino acid changes, namely A37T, Q131L, M145T, S193N, and I388S. To analyze whether MafR functions as a global transcriptional regulator, we constructed an OG1RF derivative, named OG1RF $\triangle m a f R$, in which the chromosomal region between coordinates 2421575 and 2422640 was deleted (coordinates 2889057 and 2890122 in the V583 genome; see Figure 1). This deletion removes the -10 hexamer of the Pma promoter and the first 344 codons of the mafR gene but not the IR element. Therefore, strain OG1RF $\Delta$ mafR is not able to synthesize MafR.

\section{MafR Influences Transcription of Numerous Enterococcal Genes}

The OG1RF genome has 2,658 predicted genes (Bourgogne et al., 2008). By genome-wide microarrays, we obtained the transcriptional profiles of strains OG1RF and OG1RF $\Delta m a f R$ grown to mid-log phase under standard laboratory conditions (BHI broth, $37^{\circ} \mathrm{C}$, without aeration). The total number of OG1RF genes represented in the array was 2,629. In MafRlacking cells, 90 genes were significantly differentially expressed $(P$-value $<0.05)$ : 87 genes were down-regulated $\left(\log _{2} \mathrm{FC}\right.$ of mutant strain versus wild-type strain was lower than -3 ; see Supplementary Table S1), and three genes were up-regulated ( $\log _{2} \mathrm{FC}$ of mutant strain versus wild-type strain was about 4). The latter genes were OG1RF_10454 (fruA, PTS family fructose porter, IIABC component), OG1RF_10455 (fruk2, 1phosphofructokinase), and OG1RF_10456 (lacR, lactose PTS family porter repressor), which constitute an operon (lacRfruk2-fruA). Thus, MafR influences negatively the transcription of such an operon. Among the 87 down-regulated genes, 15 genes encode components of PTS-type membrane transporters and nine genes encode components of ABC-type membrane transporters. In addition, and according to KEGG (Kyoto Encyclopedia of Genes and Genomes) annotations, 18 genes encode enzymes involved in carbon source metabolism (see Supplementary Table S1). In the absence of MafR, the highest reduction in gene expression ( $\log _{2} \mathrm{FC}$ about -6) corresponded to the OG1RF_10296-98 operon, which is involved in mannitol utilization. A high reduction in gene expression ( $\log _{2} \mathrm{FC}$ about -5 ) was also found in (i) the OG1RF_11146-49 operon, involved in glycerol metabolism, (ii) the OG1RF_11753 gene, which encodes the EIIBC component of a trehalose PTS transporter, and (iii) the OG1RF_1176361 operon, which encodes components of a carbohydrate ABC transporter.

In some pathogenic streptococci and Salmonella, mutagenesis screenings identified some PTS genes as virulence factors (Turner et al., 1998; Jones et al., 2000; Hava and Camilli, 2002). Our microarray analysis showed that MafR influences positively the expression of numerous PTS genes, as mtlA2 and mltF2 (mannitol PTS transporter). Both genes and $m t l D$ constitute the OG1RF_10296-98 operon (Table 3). Gene $m t l D$ was shown to be significantly up-regulated during growth of E. faecalis in the intestinal tract of mice (Lindenstrauss et al., 2014). Among the mannose family of PTS transporters, E. faecalis encodes a gluconate specific EII system, which consists of four components (Zúñiga et al., 2005). It is part of a predicted metabolic pathway (gluconate utilization) that consists of two operons, OG1RF_12399-97 and OG1RF_12405-00. Both operons (nine genes) were down-regulated in the absence of MafR $\left(\log _{2} \mathrm{FC}\right.$ values between -4.8 and -3.7 ; Table 3 ), and were shown to be up-regulated during growth of E. faecalis in blood (Vebø et al., 2009). Furthermore, transcription of the putative operon OG1RF_11616-11, which encodes four components of another mannose-class PTS transporter, was reduced in the absence of MafR ( $\log _{2} \mathrm{FC}$ values between -4.8 and -3.3 ). Zúñiga et al. (2005) have suggested that mannose-class PTS transporters might play a significant role in the adaptation of bacteria to epithelial surfaces.

TABLE 3 | Operons down-regulated in MafR-lacking cells.

\begin{tabular}{lll}
\hline Operon & Description & $\begin{array}{l}\text { Gene validated by } \\
\text { qRT-PCR }\end{array}$ \\
\hline OG1RF_10296-98 & $\begin{array}{l}\text { Mannitol utilization } \\
\text { (PTS transporter) }\end{array}$ & OG1RF_10298 (mtID) \\
OG1RF_10683-80 & Maltose metabolism & OG1RF_10683 (malP) \\
OG1RF_11003-05 & $\begin{array}{l}\text { ABC transporter } \\
\text { (unknown substrate) }\end{array}$ & ND \\
OG1RF_11135-33 & $\begin{array}{l}\text { Sugar ABC transporter } \\
\text { Glycerol metabolism }\end{array}$ & OG1RF_11135 \\
OG1RF_11146-49 & OG1RF_11146 (g/dA) \\
OG1RF_11181-88 & $\begin{array}{l}\text { Molybdenum cofactor } \\
\text { biosynthesis (ABC }\end{array}$ & ND \\
& $\begin{array}{l}\text { transporter) } \\
\text { Glycerol metabolism }\end{array}$ & OG1RF_11592 (glpK) \\
OG1RF_11592-90 & $\begin{array}{l}\text { Mannose-class PTS } \\
\text { transporter }\end{array}$ & ND \\
OG1RF_11616-11 & OG1RF_11763 \\
OG1RF_11763-61 & $\begin{array}{l}\text { transporter } \\
\text { Selenocompound } \\
\text { metabolism } \\
\text { Gluconate utilization }\end{array}$ & OG1RF_11948 (selD) \\
OG1RF_11951-44 & OG1RF_12398 (uxuA) \\
OG1RF_12399-97 transporter) & OG1RF_12405 (gnd2) \\
OG1RF_12405-00 & OG1RF_12564 (citF) \\
OG1RF_12571-60 & Citrate utilization &
\end{tabular}

OG1RF_12572-73

$N D$, no determined. 
MafR influences positively the expression of several ABC transporters (Table 3). In addition to the OG1RF_11763-61 operon (carbohydrate $\mathrm{ABC}$ transporter), transcription of the putative operon OG1RF_11135-33 (sugar substrate) was reduced in MafR-lacking cells $\left(\log _{2} \mathrm{FC}\right.$ about -4$)$. Expression of this operon was up-regulated during growth of E. faecalis in blood (Vebø et al., 2009), and was highly induced during peritoneum infection (Muller et al., 2015). Furthermore, the OG1RF_1100305 operon $\left(\log _{2} \mathrm{FC}\right.$ about -3 ; $\mathrm{ABC}$ transporter of unknown substrate) and genes OG1RF_11186-88 (molybdenum substrate) were down-regulated in the absence of MafR. The latter genes are part of an eight-gene operon (OG1RF_11181-88) that includes genes involved in molybdenum cofactor biosynthesis. Seven genes of such an operon were differentially expressed in MafRlacking cells $\left(\log _{2}\right.$ FC between -2.5 and -3.7$)$.

OG1RF_10683 (malP) is the first gene of a four-gene operon that includes OG1RF_10682 (malB), OG1RF_10681 (malM) and OG1RF_10680 (malR). The malPBMR operon and its neighboring OG1RF_10684 gene (malT, PTS transporter) are divergently transcribed and were shown to be involved in maltose utilization (Le Breton et al., 2005). The malPBMR operon was originally designated bopABCD (biofilm on plastic surfaces) because it was found to influence biofilm formation (Hufnagel et al., 2004). A mutational analysis in this operon showed a correlation between the ability of E. faecalis to form biofilm and its ability to colonize the mouse intestinal tract (Creti et al., 2006). The malPBMR operon was found to be partly up-regulated during growth of E. faecalis in human urine (Vebø et al., 2010). Moreover, two genes of the operon (malP and malB) and malT were up-regulated during growth of E. faecalis in blood (Vebø et al., 2009). Our microarray results revealed that malP, malB, and malT were down-regulated in MafR-lacking cells $\left(\log _{2} \mathrm{FC}\right.$ about -4 ; Table 3), suggesting that MafR might play a positive role in biofilm production. Genes OG1RF_11951 (seleniumdependent molybdenum hydroxylase) and OG1RF_11948 (selD; selenide, water dikinase) were also down-regulated in the absence of MafR $\left(\log _{2}\right.$ FC about -3$)$. Both genes, which form part of a putative eight-gene operon (OG1RF_11951-44 operon), were reported to contribute positively to biofilm formation (Ballering et al., 2009; Srivastava et al., 2011).

Enterococcus faecalis is able to use citrate as the sole source of carbon and energy. Citrate metabolism has been extensively investigated in this bacterium (Blancato et al., 2008; Suárez et al., 2011; Repizo et al., 2013). The cit locus is constituted by two divergent operons: citHO and oadHDB-citCDEFX-oadAcitMG. Our microarray analysis revealed that the cit locus (OG1RF_12572-73 and OG1RF_12571-60) was down-regulated in the absence of MafR (Table 3). With the exception of citO (OG1RF_12573; $\left.\log _{2} \mathrm{FC}-1.66\right)$ and citG (OG1RF_12560; $\left.\log _{2} \mathrm{FC}-2.46\right)$, the $\log _{2} \mathrm{FC}$ value of the cit genes was between 5.38 and -3.07 (see Supplementary Table S1). Therefore, MafR has a positive effect on the transcription of the two operons involved in citrate utilization. Several genes of the cit locus were up-regulated during growth of E. faecalis in human urine (Vebø et al., 2010). Furthermore, genes responsible for citrate utilization were found to be up-regulated during the adaptation of E. faecalis to blood (Vebø et al., 2009).
Glycerol can be a carbon/energy source for several pathogenic bacteria (Ramsey et al., 2014). Two pathways for glycerol catabolism are present in E. faecalis, named GlpO/GlpK and GldA/DhaK. The $g l p K, g l p O$, and $g l p F$ genes (OG1RF_1159290) constitute an operon. Our microarray analysis showed that this operon was down-regulated in MafR-lacking cells $\left(\log _{2} \mathrm{FC}\right.$ about -3). Also, the OG1RF_11146-49 operon, which includes genes gld $A$ and $d h a K$, was down-regulated $\left(\log _{2} \mathrm{FC}\right.$ about -5 ; Table 3). Hence, MafR influences positively the transcription of both glycerol catabolic operons, although the activator effect seems to be greater on the expression of the GldA/DhaK pathway. Both operons were up-regulated during the adaptation of $E$. faecalis to the intestinal tract of mice (Lindenstrauss et al., 2014), and also during mice peritonitis (Muller et al., 2015). Moreover, enterococcal mutant strains unable to metabolize glycerol were affected in organ colonization in a systemic murine infection model (Muller et al., 2015). Vebø et al. (2009) reported that the $g l p K O F$ operon is highly up-regulated during growth of $E$. faecalis in blood. We hypothesize that MafR may facilitate the survival of $E$. faecalis in particular host niches through the transcriptional activation of the glycerol catabolic operons.

Many eukaryotic proteins are glycosylated, including some proteins of the immune system (Apweiler et al., 1999). Proteolytic and glycolytic modulation of the host immune system appears to be a common theme in the pathogenesis of some Gram-positive bacteria (Collin and Olsén, 2003). E. faecalis encodes several putative glycosidases but little is known about their biological function. The endo- $\beta-N$ acetylglucosaminidase EndoE (OG1RF_10107) was shown to cleave the N-linked glycans of both the human immunoglobulin G (Collin and Fischetti, 2004) and the human glycoprotein lactoferrin (Garbe et al., 2014), which is considered to be a component of innate immunity. Gene OG1RF_12167 also encodes an endo- $\beta-N$-acetylglucosaminidase activity (Bøhle et al., 2011). Our microarray analysis revealed that transcription of both genes (OG1RF_10107 and OG1RF_12167) was highly reduced in the absence of MafR $\left(\log _{2} \overline{F C}\right.$ about -4 ; see Supplementary Table S1).

\section{Validation of the Microarray Results by qRT-PCR}

To validate the microarray results, we performed qRT-PCR assays using the comparative $C_{\mathrm{T}}$ method (Schmittgen and Livak, 2008). Specifically, we selected 12 potential MafR target genes involved in metabolism ( $m t l D$, malP, gldA, glpK, selD, uxuA, gnd2, citF), regulation (lacR) or transport (malT, OG1RF_11135, OG1RF_11763; Table 3). These genes are transcribed from different promoters. We determined their relative expression in OG1RF and OG1RF $\triangle m a f R$ using recA as internal control gene (Figure 5). Except for lacR, gene expression was reduced in strain OG1RF $\triangle$ mafR compared to OG1RF. In all the cases, the fold change in gene expression $\left(\log _{2} \mathrm{FC}\right)$ due to the lack of MafR (OG1RF $\triangle$ mafR versus OG1RF) was comparable to the value obtained in the microarray experiments (Figure 5). Additionally, we performed qRT-PCR assays using total RNA isolated from strains $\mathrm{JH} 2-2$ and $\mathrm{JH} 2-2 \Delta m a f R$. In this case, 


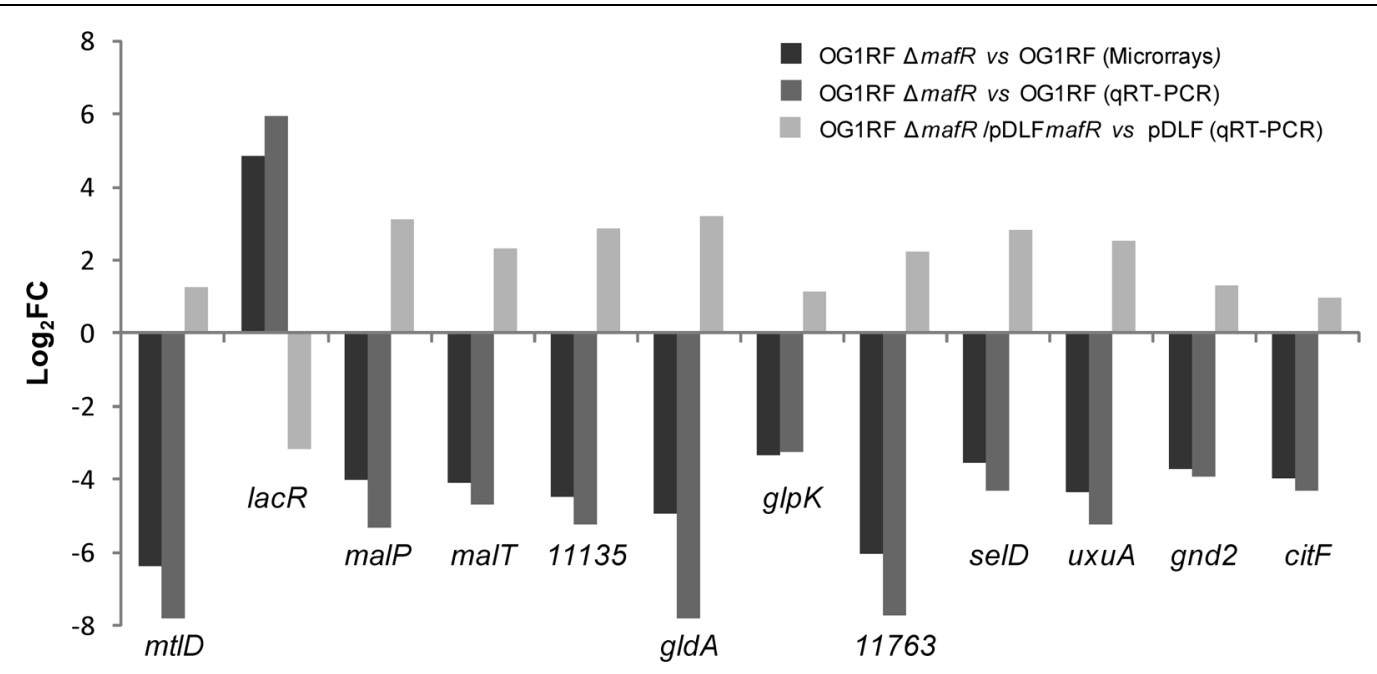

FIGURE 5 | Effect of MafR on gene expression. $\log _{2}$ fold change in the expression of the indicated genes due to the lack of MafR (OG1RF $\Delta$ mafR vs. OG1RF) determined by microarray analysis and by qRT-PCR. Log $_{2}$ fold change in gene expression due to the presence of the MafR-encoding plasmid (OG1RF $\Delta$ mafR/pDLFmafR vs. OG1RF $\triangle$ mafR/pDLF).

we determined the relative expression of genes lack, $m t l D$, gldA, and citF. The results obtained confirmed that MafR has a negative effect on the transcription of lack but a positive effect on the transcription of $m t l D, g l d A$, and citF (not shown).

\section{Genetic Complementation Studies in Strain OG1RF $\Delta$ mafR}

For genetic complementation of the mafR deletion, we inserted the mafR gene into the multicopy pDLF expression vector (this work), and introduced the pDLFmafR recombinant plasmid into the mafR deletion mutant strain (OG1RF $\Delta$ mafR). By qRT-PCR, we determined the relative expression of the 12 genes mentioned above in both strains: OGIRF $\triangle$ mafR harboring pDLFmafR (plasmid-encoded MafR) and OG1RF $\triangle m a f R$ harboring pDLF (absence of MafR). The fold change in gene expression $\left(\log _{2} \mathrm{FC}\right.$ ) due to plasmid-encoded MafR is shown in Figure 5. Compared to cells harboring pDLF, gene lacR was down-regulated in cells harboring pDLFmafR. On the contrary, the other 11 genes were up-regulated. Hence, plasmid-encoded MafR increases the transcription of the 11 chromosomal genes. Nevertheless, such an increase is lower than the one due to the single chromosomal copy of mafR (see OG1RF $\Delta$ mafR versus OG1RF in Figure 5). This result suggests that only part of the plasmid-encoded MafR protein is active.

\section{Potential Curvatures in Promoter Regions of MafR-Regulated Operons}

MafR is thought to be a member of the Mga/AtxA family of global response regulators. These regulators appear to bind DNA with low sequence specificity. Sequence alignments of all established Mga-binding regions revealed that they exhibit only $13.4 \%$ identity (Hause and McIver, 2012). In the case of AtxA, in silico analyses revealed that the promoter regions of several target genes are intrinsically curved (Hadjifrangiskou and Koehler, 2008). Furthermore, in vitro studies showed that MgaSpn interacts with DNA sites that contain a potential intrinsic curvature (Solano-Collado et al., 2013). Recently, Innocenti et al. (2015) mapped numerous transcription start sites on the V583 genome using a modified RNA-seq approach. Their study allowed us to locate the transcription start sites of six MafR-regulated operons on the OG1RF genome. The first gene of each operon and its transcription start site are listed in Table 4. We calculated the curvature propensity plots of the corresponding promoter regions (positions -1 to -200) using the bend.it program (Vlahovicek et al., 2003). Compared with the global $\mathrm{A}+\mathrm{T}$ content (62.2\%) of the OG1RF genome, five out of the six promoter regions display a high $\mathrm{A}+\mathrm{T}$ content $(74-77 \%)$. Moreover, they contain one peak of potential

TABLE 4 | Potential intrinsic curvatures within promoter regions.

\begin{tabular}{lllll}
\hline Locus tag (gene) & $\begin{array}{l}\text { TSS } \\
\text { position }\end{array}$ & $\begin{array}{l}\mathbf{A}+\mathbf{T}^{\mathbf{b}} \\
(\%)\end{array}$ & $\begin{array}{l}\text { Curvature }^{\mathbf{c}} \\
\text { (\%/helical turn) }\end{array}$ & Location $^{\mathbf{d}}$ \\
\hline OG1RF_10296 (mt/A2) & 310152 & 64.5 & 12.02 & -40 \\
OG1RF_11146 (g/d/A) & 1197479 & 75.5 & 13.16 & -179 \\
& & & 16.16 & -63 \\
OG1RF_11592 (g/pK) & 1660384 & 77 & 17.35 & -171 \\
OG1RF_11763 & 1846229 & 74.5 & 14.64 & -86 \\
& & & 15.01 & -78 \\
OG1RF_12405 (gnd2) & 2542744 & 74 & 12.92 & -136 \\
& & & 14.27 & -76 \\
OG1RF_12571 & 2733071 & 76.5 & 14.83 & -85 \\
& & & 13.71 & -24 \\
\hline
\end{tabular}

a Position of the transcription start site on the OG1RF genome.

${ }^{\mathrm{b}} A+T$ content of the promoter region (from position -1 to position -200).

${ }^{c}$ Predicted curvatures with a magnitude $>12$ degrees $/ 10.5$ bp helical turn.

d Location of peaks of potential curvatures within the promoter regions (relative to the TSS). 

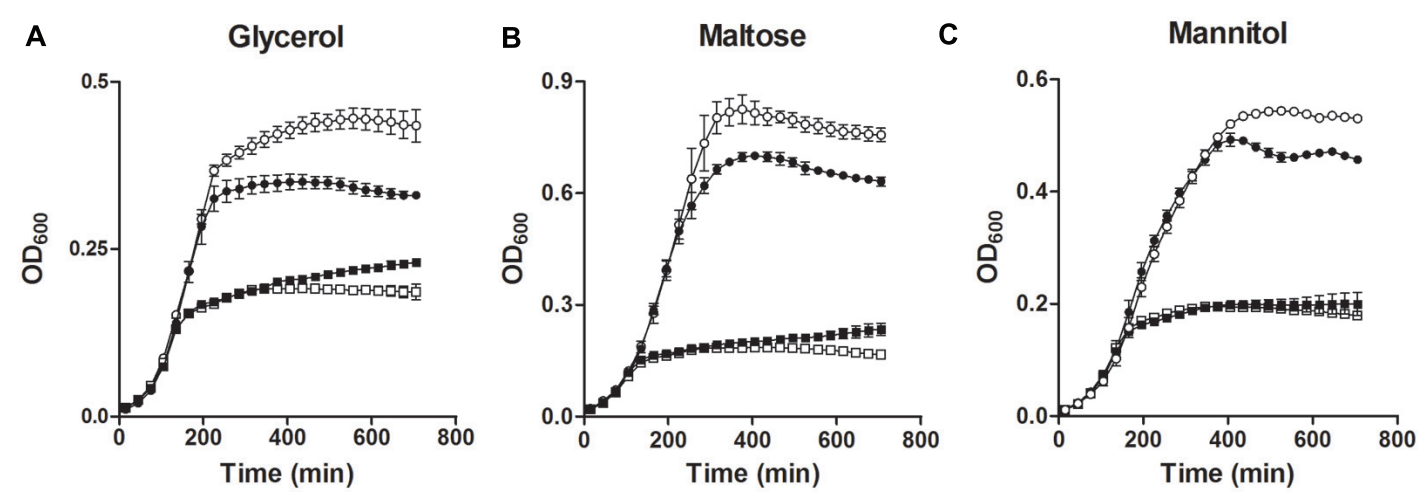

FIGURE 6 | Effect of MafR on the utilization of different carbon sources. Bacteria were grown in M9YE medium supplemented (circles) or not (squares) with glycerol (A), maltose (B), or mannitol (C). White symbols correspond to strain OG1RF. Black symbols correspond to strain OG1RF $\Delta$ mafR. Data correspond to the mean values of three independent experiments. Error bars indicate standard error of mean.

sequence-dependent curvature with a magnitude higher than 14 degrees per helical turn. The peak of such a curvature is located at position -171 in gene $g l p K$, and between positions -63 and -86 in genes gldA, OG1RF_11763, gnd2, and OG1RF_12571 (Table 4). Further work is required to determine whether MafR is able to bind to DNA containing such potential intrinsic curvatures.

\section{MafR Plays a Positive Role in the Utilization of Different Carbon Sources}

Two operons for glycerol metabolism are present in E. faecalis. Both of them were down-regulated in MafR-lacking cells (Table 3, Figure 5). To evaluate the effect of MafR on the utilization of glycerol, we analyzed the growth of strains OG1RF and OG1RF $\triangle m a f R$ in M9YE medium supplemented or not with glycerol (Figure 6A). In the absence of glycerol, both cultures reached a similar $\mathrm{OD}_{600}$ (about 0.2). However, in glycerol-supplemented medium, the final $\mathrm{OD}_{600}$ reached in cultures of the mutant strain was lower than that attained in cultures of the wild-type strain. Furthermore, compared to the wild-type strain, the MafR-lacking strain showed a diminished growth in M9YE medium supplemented with maltose (Figure 6B), which correlates with the lower expression found in genes malP, malB, and malT (maltose utilization; Table 3, Figure 5). The growth of the mutant strain was also reduced in M9YE medium supplemented with mannitol (Figure 6C), which is linked to the reduction in expression of the OG1RF_10296-98 operon (mannitol utilization; Table 3, Figure 5). Therefore, MafR has a positive effect on the utilization of glycerol, maltose, and mannitol.

\section{MafR Influences the Host Inflammatory Response During Mice Peritonitis}

To investigate the influence of the MafR regulator in vivo, a peritonitis infection model was used comparing the mafR deletion mutant strain (OG1RF $\triangle m a f R$ ) and the corresponding isogenic wild-type strain (OG1RF). Our results suggest a role

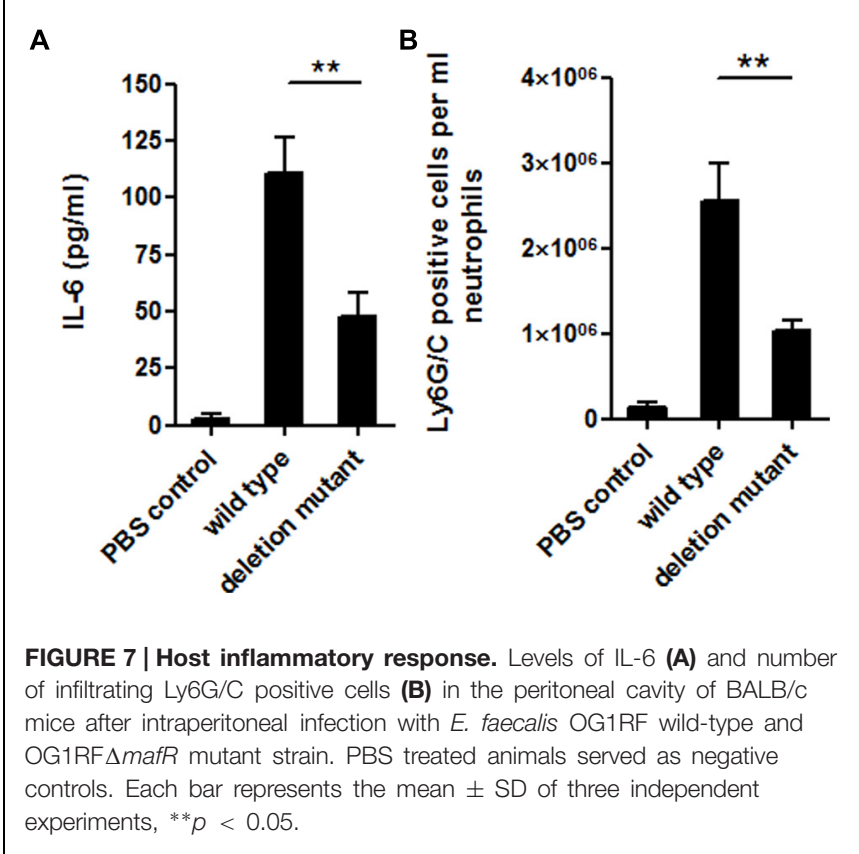

of MafR in bacterial virulence. A significant higher degree of inflammation could be obtained in the peritoneal cavity of mice infected with the wild-type bacteria compared to the mafR deletion mutant. This finding was indicated on the cytokine level by a higher release of IL-6 (Figure 7A), an important systemic inflammatory cytokine, and on the cellular basis by higher numbers of infiltrating neutrophils (Figure 7B) to the site of infection. It is important to state that even though the number of infiltrating neutrophils was 2.5 times higher if mice were infected with the wildtype bacteria compared to the mutant strain, no significant reduction in the bacterial loads within the peritoneal cavity could be observed (data not shown); this also highlights the possible role of the MafR regulator in bacterial virulence. Taken together, our observations clearly suggest an in vivo role 
of the MafR regulator in the course of infections caused by E. faecalis. The precise underlying molecular mechanisms of how this regulatory axis is contributing to bacterial virulence, however, need to be elucidated in further studies.

\section{CONCLUSION}

Our understanding of the mechanisms involved in the pathogenicity of E. faecalis is still very limited. In this work, we have constructed a deletion mutant strain to investigate the function of the mafR gene. By genomewide microarrays, quantitative RT-PCR assays, and complementation studies, we have shown that MafR increases the expression of numerous genes. The most significant target genes are involved in the utilization of carbon sources. Associated to this fact, we have found that the growth of the mafR deletion mutant strain is impaired in media containing glycerol, maltose, or mannitol. In addition, we have shown that enterococcal cells deficient in MafR induce a more moderate inflammatory response in a mouse peritonitis model. We propose that MafR facilitates the adaptation of E. faecalis to particular host niches and, consequently, it contributes to its potential virulence.

\section{REFERENCES}

Apweiler, R., Hermjakob, H., and Sharon, N. (1999). On the frequency of protein glycosylation as deduced from analysis of the SWISS-PROT database. BBA-Gen. Subjects 1473, 4-8. doi: 10.1016/S0304-4165(99)00165-8

Ballering, K. S., Kristich, C. J., Grindle, S. M., Oromendia, A., Beattie, D. T., and Dunny, G. M. (2009). Functional genomics of Enterococcus faecalis: multiple novel genetic determinants for biofilm formation in the core genome. J. Bacteriol. 191, 2806-2814. doi: 10.1128/JB.01688-08

Blancato, V. S., and Magni, C. (2010). A chimeric vector for efficient chromosomal modification in Enterococcus faecalis and other lactic acid bacteria. Lett. Appl. Microbiol. 50, 542-546. doi: 10.1111/j.1472-765X.2010.02815.X

Blancato, V. S., Repizo, G. D., Suárez, C. A., and Magni, C. (2008). Transcriptional regulation of the citrate gene cluster of Enterococcus faecalis involves the GntR family transcriptional activator CitO. J. Bacteriol. 190, 7419-7430. doi: 10.1128/JB.01704-07

Bøhle, L. A., Mathiesen, G., Vaaje-Kolstad, G., and Eijsink, V. G. (2011). An endo- $\beta-\mathrm{N}$-acetylglucosaminidase from Enterococcus faecalis V583 responsible for the hydrolysis of high-mannose and hybrid-type N-linked glycans. FEMS Microbiol. Lett. 325, 123-129. doi: 10.1111/j.1574-6968.2011. 02419.x

Bourgogne, A., Drysdale, M., Hilsenbeck, S. G., Peterson, S. N., and Koehler, T. M. (2003). Global effects of virulence gene regulators in a Bacillus anthracis strain with both virulence plasmids. Infect. Immun. 71, 2736-2743. doi: 10.1128/IAI.71.5.2736-2743.2003

Bourgogne, A., Garsin, D. A., Qin, X., Singh, K. V., Sillanpaa, J., Yerrapragada, S., et al. (2008). Large scale variation in Enterococcus faecalis illustrated by the genome analysis of strain OG1RF. Genome Biol. 9, R110-R110. doi: 10.1186/gb2008-9-7-r110

Collin, M., and Fischetti, V. A. (2004). A novel secreted endoglycosidase from Enterococcus faecalis with activity on human immunoglobulin $G$ and ribonuclease B. J. Biol. Chem. 279, 22558-22570. doi: 10.1074/jbc.M402156200

Collin, M., and Olsén, A. (2003). Extracellular enzymes with immunomodulating activities: variations on a theme in Streptococcus pyogenes. Infect. Immun. 71, 2983-2992. doi: 10.1128/IAI.71.6.2983-2992.2003

\section{AUTHOR CONTRIBUTIONS}

SR-C, ME, OG, and AB designed the study. SR-C and OG performed laboratory work. SR-C, ME, OG, and AB performed data analysis and wrote the manuscript. All authors read and approved the final manuscript.

\section{ACKNOWLEDGMENTS}

Thanks are due to Verónica Navarro for her excellent technical assistance, and to Dr. Christian Magni for providing the pBVGh plasmid. This work was supported by grants CSD2008-00013INTERMODS to ME and BIO2013-49148-C2-2-R to AB from the Spanish Ministry of Economy and Competitiveness, CSIC-PIE201320 E028 to AB from the Spanish National Research Council. SR-C was a recipient of a fellowship (AP2008-00105) from the Spanish Ministry of Education.

\section{SUPPLEMENTARY MATERIAL}

The Supplementary Material for this article can be found online at: http://journal.frontiersin.org/article/10.3389/fmicb. 2015.01521

Creti, R., Koch, S., Fabretti, F., Baldassarri, L., and Huebner, J. (2006). Enterococcal colonization of the gastro-intestinal tract: role of biofilm and environmental oligosaccharides. BMC Microbiol. 6:60. doi: 10.1186/1471-2180-6-60

Deutscher, J., Aké, F. M. D., Derkaoui, M., ZéBré, A. C., Cao, T. N., Bouraoui, H., et al. (2014). The bacterial phosphoenolpyruvate:carbohydrate phosphotransferase system: regulation by protein phosphorylation and phosphorylation-dependent protein-protein interactions. Microbiol. Mol. Biol. Rev. 78, 231-256. doi: 10.1128/MMBR.00001-14

Edgar, R., Domrachev, M., and Lash, A. E. (2002). Gene Expression Omnibus: NCBI gene expression and hybridization array data repository. Nucleic Acids Res. 30, 207-210. doi: 10.1093/nar/30.1.207

Finn, R. D., Tate, J., Mistry, J., Coggill, P. C., Sammut, S. J., Hotz, H.-R., et al. (2008). The Pfam protein families database. Nucleic Acids Res. 36, D281-D288. doi: $10.1093 / \mathrm{nar} / \mathrm{gkm} 960$

Fisher, K., and Phillips, C. (2009). The ecology, epidemiology and virulence of Enterococcus. Microbiology 155, 1749-1757. doi: 10.1099/mic.0.026385-0

Garbe, J., Sjögren, J., Cosgrave, E. F. J., Struwe, W. B., Bober, M., Olin, A. I., et al. (2014). EndoE from Enterococcus faecalis hydrolyzes the glycans of the biofilm inhibiting protein lactoferrin and mediates growth. PLOS ONE 9:e91035. doi: 10.1371/journal.pone.0091035

Hadjifrangiskou, M., and Koehler, T. M. (2008). Intrinsic curvature associated with the coordinately regulated anthrax toxin gene promoters. Microbiology 154, 2501-2512. doi: 10.1099/mic.0.2007/016162-0

Hammerstrom, T. G., Horton, L. B., Swick, M. C., Joachimiak, A., Osipiuk, J., and Koehler, T. M. (2015). Crystal structure of Bacillus anthracis virulence regulator AtxA and effects of phosphorylated histidines on multimerization and activity. Mol. Microbiol. 95, 426-441. doi: 10.1111/mmi.12867

Hause, L. L., and McIver, K. S. (2012). Nucleotides critical for the interaction of the Streptococcus pyogenes Mga virulence regulator with Mga-regulated promoter sequences. J. Bacteriol. 194, 4904-4919. doi: 10.1128/JB.00809-12

Hava, D. L., and Camilli, A. (2002). Large-scale identification of serotype 4 Streptococcus pneumoniae virulence factors. Mol. Microbiol. 45, 1389-1406. doi: 10.1046/j.1365-2958.2002.03106.x

Hemsley, C., Joyce, E., Hava, D. L., Kawale, A., and Camilli, A. (2003). MgrA, an orthologue of Mga, acts as a transcriptional repressor of the genes within 
the rlrA pathogenicity islet in Streptococcus pneumoniae. J. Bacteriol. 185, 6640-6647. doi: 10.1128/JB.185.22.6640-6647.2003

Hollenbeck, B. L., and Rice, L. B. (2012). Intrinsic and acquired resistance mechanisms in enterococcus. Virulence 3, 421-469. doi: 10.4161/viru.21282

Hondorp, E. R., Hou, S. C., Hause, L. L., Gera, K., Lee, C.-E., and McIver, K. S. (2013). PTS phosphorylation of Mga modulates regulon expression and virulence in the group A streptococcus. Mol. Microbiol. 88, 1176-1193. doi: 10.1111/mmi. 12250

Hondorp, E. R., and McIver, K. S. (2007). The Mga virulence regulon: infection where the grass is greener. Mol. Microbiol. 66, 1056-1065. doi: 10.1111/j.13652958.2007.06006.x

Hufnagel, M., Koch, S., Creti, R., Baldassarri, L., and Huebner, J. (2004). A putative sugar-binding transcriptional regulator in a novel gene locus in Enterococcus faecalis contributes to production of biofilm and prolonged bacteremia in mice. J. Infect. Dis. 189, 420-430. doi: 10.1086/381150

Innocenti, N., Golumbeanu, M., d'Hérouël, A., Lacoux, C., Bonnin, R. A., Kennedy, S. P., et al. (2015). Whole-genome mapping of 5' RNA ends in bacteria by tagged sequencing: a comprehensive view in Enterococcus faecalis. RNA 21, 1018-1030. doi: $10.1261 /$ rna.048470.114

Jacob, A. E., and Hobbs, S. J. (1974). Conjugal transfer of plasmid-borne multiple antibiotic resistance in Streptococcus faecalis var. zymogenes. J. Bacteriol. 117, 360-372.

Jones, A. L., Knoll, K. M., and Rubens, C. E. (2000). Identification of Streptococcus agalactiae virulence genes in the neonatal rat sepsis model using signaturetagged mutagenesis. Mol. Microbiol. 37, 1444-1455. doi: 10.1046/j.13652958.2000.02099.x

LeBlanc, D. J., Chen, Y. Y., and Lee, L. N. (1993). Identification and characterization of a mobilization gene in the streptococcal plasmid, pVA380-1. Plasmid 30, 296-302. doi: 10.1006/plas.1993.1063

Le Breton, Y., Pichereau, V., Sauvageot, N., Auffray, Y., and Rincé, A. (2005). Maltose utilization in Enterococcus faecalis. J. Appl. Microbiol. 98, 806-813. doi: 10.1111/j.1365-2672.2004.02468.x

Lindenstrauss, A. G., Ehrmann, M. A., Behr, J., Landstorfer, R., Haller, D., Sartor, R. B., et al. (2014). Transcriptome analysis of Enterococcus faecalis toward its adaption to surviving in the mouse intestinal tract. Arch. Microbiol. 196, 423-433. doi: 10.1007/s00203-014-0982-2

Muller, C., Cacaci, M., Sauvageot, N., Sanguinetti, M., Rattei, T., Eder, T., et al. (2015). The intraperitoneal transcriptome of the opportunistic pathogen Enterococcus faecalis in mice. PLoS ONE 10:e0126143. doi: 10.1371/journal.pone.0126143

Paulsen, I. T., Banerjei, L., Myers, G. S. A., Nelson, K. E., Seshadri, R., Read, T. D., et al. (2003). Role of mobile DNA in the evolution of vancomycinresistant Enterococcus faecalis. Science 299, 2071-2074. doi: 10.1126/science. 1080613

Poncet, S., Milohanic, E., Mazé, A., Abdallah, J. N., Aké, F., Larribe, M., et al. (2009). Correlations between carbon metabolism and virulence in bacteria. Collin M, Schuch R (eds): bacterial Sensing and Signaling. Contrib. Microbiol. Basel Karger 16, 88-102. doi: 10.1159/000219374

Ramsey, M., Hartke, A., and Huycke, M. (2014). "The physiology and metabolism of Enterococci," in Enterococci: From Commensals to Leading Causes of Drug Resistant Infection (Internet), eds M. S. Gilmore, D. B. Clewell, Y. Ike, et al. (Boston: Massachusetts Eye and Ear Infirmary), 1-43.

Repizo, G. D., Blancato, V. S., Mortera, P., Lolkema, J. S., and Magni, C. (2013). Biochemical and genetic characterization of the Enterococcus faecalis oxaloacetate decarboxylase complex. Appl. Environ. Microb. 79, 2882-2890. doi: 10.1128/AEM.03980-12

Ribardo, D. A., and McIver, K. S. (2006). Defining the Mga regulon: comparative transcriptome analysis reveals both direct and indirect regulation by Mga in the group A streptococcus. Mol. Microbiol. 62, 491-508. doi: 10.1111/j.13652958.2006.05381.x

Rice, P., Longden, I., and Bleasby, A. (2000). EMBOSS: the european molecular biology open software suite. Trends Genet. 16, 276-277. doi: 10.1016/S01689525(00)02024-2
Ruiz-Cruz, S., Solano-Collado, V., Espinosa, M., and Bravo, A. (2010). Novel plasmid-based genetic tools for the study of promoters and terminators in Streptococcus pneumoniae and Enterococcus faecalis. J. Microbiol. Methods 83, 156-163. doi: 10.1016/j.mimet.2010.08.004

Sanson, M., Makthal, N., Gavagan, M., Cantu, C., Olsen, R. J., Musser, J. M., et al. (2015). Phosphorylation events in the multiple gene regulator of group a Streptococcus significantly influence global gene expression and virulence. Infect. Immun. 83, 2382-2395. doi: 10.1128/IAI.03023-14

Schmittgen, T. D., and Livak, K. J. (2008). Analyzing real-time PCR data by the comparative CT method. Nat. Protoc. 3, 1101-1108. doi: 10.1038/nprot.2008.73

Shepard, B. D., and Gilmore, M. S. (1995). Electroporation and efficient transformation of Enterococcus faecalis grown in high concentrations of glycine. Methods Mol. Biol. 47, 217-226.

Solano-Collado, V., Espinosa, M., and Bravo, A. (2012). Activator role of the pneumococcal Mga-like virulence transcriptional regulator. J. Bacteriol. 194, 4197-4207. doi: 10.1128/JB.00536-12

Solano-Collado, V., Lurz, R., Espinosa, M., and Bravo, A. (2013). The pneumococcal MgaSpn virulence transcriptional regulator generates multimeric complexes on linear double-stranded DNA. Nucleic Acids Res. 41, 6975-6991. doi: 10.1093/nar/gkt445

Srivastava, M., Mallard, C., Barke, T., Hancock, L. E., and Self, W. T. (2011). A selenium-dependent xanthine dehydrogenase triggers biofilm proliferation in Enterococcus faecalis through oxidant production. J. Bacteriol. 193, 1643-1652. doi: 10.1128/JB.01063-10

Suárez, C., Blancato, V., Poncet, S., Deutscher, J., and Magni, C. (2011). CcpA represses the expression of the divergent cit operons of Enterococcus faecalis through multiple cre sites. BMC Microbiol. 11:227. doi: 10.1186/1471-2180$11-227$

Tsvetanova, B., Wilson, A. C., Bongiorni, C., Chiang, C., Hoch, J. A., and Perego, M. (2007). Opposing effects of histidine phosphorylation regulate the AtxA virulence transcription factor in Bacillus anthracis. Mol. Microbiol. 63, 644-655. doi: 10.1111/j.1365-2958.2006.05543.x

Turner, A. K., Lovell, M. A., Hulme, S. D., Zhang-Barber, L., and Barrow, P. A. (1998). Identification of Salmonella typhimurium genes required for colonization of the chicken alimentary tract and for virulence in newly hatched chicks. Infect. Immun. 66, 2099-2106.

Vebø, H. C., Snipen, L., Nes, I. F., and Brede, D. A. (2009). The transcriptome of the nosocomial pathogen Enterococcus faecalis V583 reveals adaptive responses to growth in blood. PLoS ONE 4:e7660. doi: 10.1371/journal.pone.0007660

Vebø, H. C., Solheim, M., Snipen, L., Nes, I. F., and Brede, D. A. (2010). Comparative genomic analysis of pathogenic and probiotic Enterococcus faecalis isolates, and their transcriptional responses to growth in human urine. PLOS ONE 5:e12489. doi: 10.1371/journal.pone.0012489

Vlahovicek, K., Kaján, L., and Pongor, S. (2003). DNA analysis servers: plot.it, bend.it, model.it and IS. Nucleic Acids Res. 31, 3686-3687. doi: 10.1093/nar/gkg559

Yanisch-Perron, C., Vieira, J., and Messing, J. (1985). Improved M13 phage cloning vectors and host strains: nucleotide sequences of the M13mp18 and pUC19 vectors. Gene 33, 103-119. doi: 10.1016/0378-1119(85)90120-9

Zúñiga, M., Comas, I., Linaje, R., Monedero, V., Yebra, M. J., Esteban, C. D., et al. (2005). Horizontal gene transfer in the molecular evolution of mannose PTS transporters. Mol. Biol. Evol. 22, 1673-1685. doi: 10.1093/molbev/msi163

Conflict of Interest Statement: The authors declare that the research was conducted in the absence of any commercial or financial relationships that could be construed as a potential conflict of interest.

Copyright () 2016 Ruiz-Cruz, Espinosa, Goldmann and Bravo. This is an open-access article distributed under the terms of the Creative Commons Attribution License (CC BY). The use, distribution or reproduction in other forums is permitted, provided the original author(s) or licensor are credited and that the original publication in this journal is cited, in accordance with accepted academic practice. No use, distribution or reproduction is permitted which does not comply with these terms. 